\title{
EXISTENCE OF OPTIMAL CONTROLS FOR SEMILINEAR ELLIPTIC EQUATIONS WITHOUT CESARI-TYPE CONDITIONS
}

\author{
HONGWEI LOU'
}

(Received 27 October, 2000; revised 17 July, 2001)

\begin{abstract}
Optimal control problems governed by semilinear elliptic partial differential equations are considered. No Cesari-type conditions are assumed. By proving an existence theorem and the Pontryagin maximum principle of optimal "state-control" pairs for the corresponding relaxed problems, we establish an existence theorem of optimal pairs for the original problem.
\end{abstract}

\section{Introduction}

It is well-known to researchers working in optimal control theory that to guarantee the existence of (classical) optimal pairs we need a Cesari-type condition, which is a natural generalisation of optimal control problems with linear state equations and convex cost functionals. Many results are available along these lines. We refer the reader to the books by Berkovitz [3], Cesari [5] and $\mathrm{Li}$ and Yong [11] for further detail.

When these types of conditions are no longer satisfied, measure-valued controls (that is, randomising controls), called "relaxed controls", are introduced. Other names have been used in the literature for relaxed controls such as "sliding regimes" (Filippov [7]), "generalised controls" (Gamkrelidze [8]), "relaxed curves" (Warga [18]), and "generalised curves" (Young [22]). Here we adopt the name "relaxed control" (McShane [13]) since it is more frequently used among mathematicians working in control theory. For finite-dimensional control systems, relaxed controls have been systematically studied. We refer the reader to the books of Gamkrelidze [8], Berkovitz [3] and Warga [20] for details. For infinite-dimensional systems, most results are concerned with linear or semilinear evolution systems. Among them, we mention the works by Ahmed [1], Fattorini [6] and Papageorgiou [16].

\footnotetext{
${ }^{1}$ Mathematical Department, Fudan University, Shanghai, 200433, China; e-mail: hwlou@fudan.edu.cn. (C) Australian Mathematical Society 2003, Serial-fee code 1446-1811/03
} 
By relaxation, the space of admissible controls is extended to a larger space and both control system and cost functional are convexified. There are at least three advantages in considering relaxed controls. First, after relaxation, the existence theorem and the necessary conditions of optimal relaxed control follow easily under relatively weak hypotheses. Next, when the classical control problem (that is, non-relaxed problem) admits no optimal control, optimal relaxed control provides a method to construct approximate optimal controls since under suitable conditions, any relaxed control can be approximated by classical controls. For infinite-dimensional systems, especially for evolution systems, the above two aspects have been carefully investigated by many researchers. The third advantage is that when an optimal relaxed control is a Dirac measure almost everywhere, then it essentially becomes a classical optimal control, that is, in the non-relaxed sense (see Section 2 for details). Thus research on optimal relaxed control also gives the possibility of seeking classical optimal controls (see Balder [2], Neustadt [15] and Suryanarayana [17], for examples).

The main purpose of this paper is to establish an existence theorem for some systems governed by semilinear elliptic equations without assuming Cesari-type conditions. To this end, we first establish existence and the Pontryagin maximum principle for optimal relaxed controls. Under suitable assumptions, we can prove that an optimal relaxed control is supported at a single point almost everywhere. Thus it must be an optimal control for the classical non-relaxed problem.

\section{Classical and relaxed controls}

The (classical) control system we consider in this paper is

$$
\left\{\begin{array}{l}
-\sum_{i, j=1}^{n} \frac{\partial}{\partial x_{i}}\left(a_{i j}(x) \frac{\partial y}{\partial x_{j}}(x)\right)=f(x, y(x), u(x)), \quad \text { in } \Omega, \\
\left.y\right|_{\partial \Omega}=0,
\end{array}\right.
$$

with the cost functional being

$$
J(u(\cdot))=\int_{\Omega} f^{0}(x, y(x), u(x)) d x,
$$

where $y(\cdot)$ is the state corresponding to control $u(\cdot)$ satisfying $(2.1)$.

We pose the following assumptions.

(S1) $\Omega$ is a bounded region in $\mathbb{R}^{n}$ with a $C^{1,1}$ boundary $\partial \Omega$, and $U$ is a compact metric space.

(S2) $a_{i j}(\cdot) \in C(\bar{\Omega}), a_{i j}=a_{j i}$ and for some $\lambda>0, \sum_{i, j=1}^{n} a_{i j}(x) \xi_{i} \xi_{j} \geq \lambda|\xi|^{2}$, $\forall \xi \equiv\left(\xi_{1}, \xi_{2}, \ldots, \xi_{n}\right) \in \mathbb{R}^{n}, x \in \Omega$. 
(S3) $f: \Omega \times \mathbb{B} \times U \rightarrow \mathbb{R}$ is Borel measurable in $(x, y, u) \in \Omega \times \mathbb{R} \times U$ and continuous in $(y, u) \in \mathbb{R} \times U$ for almost all $x \in \Omega, f_{y}(x, y, u) \leq 0, \forall(x, y, u) \in$ $\Omega \times \mathbb{B} \times U$. Moreover, for any $R>0$, there exists an $M_{R}>0$ such that $|f(x, y, u)|+$ $\left|f_{y}(x, y, u)\right| \leq M_{R}, \forall(x, u) \in \Omega \times U,|y| \leq R$.

(S4) $f^{0}: \Omega \times \mathbb{R} \times U \rightarrow \mathbb{R}$ is Borel measurable in $(x, y, u)$ and lower semicontinuous in $(y, u)$ for almost all $x \in \Omega$. Moreover, for any $R>0$, there exists an $K_{R}>0$ such that $f^{0}(x, y, u) \geq-K_{R}, \forall(x, u) \in \Omega \times U,|y| \leq R$.

Denote $\mathscr{U}_{\mathrm{ad}} \triangleq\{v: \Omega \rightarrow U \mid v$ measurable $\}$. Our (classical) optimal control problem is as follows.

PROBLEM (C). Find a $\bar{u}(\cdot) \in \mathscr{U}_{\mathrm{ad}}$ such that

$$
J(\bar{u}(\cdot))=\inf _{u(\cdot) \in \mathscr{U}_{\alpha d}} J(u(\cdot)) .
$$

Any $\bar{u}(\cdot)$ satisfying (2.3) is called an optimal control. For convenience, we call it a classical optimal control. It is well-known that optimal control of Problem (C) may fail to exist unless further suitable Cesari-type conditions are imposed on $\left(f^{0}, f\right)$.

EXAMPLE 1. Let $U=[-1,1], \Omega=(-1,1)$,

$$
\begin{gathered}
\left\{\begin{array}{r}
-y^{\prime \prime}(x)=u(x), \quad \text { in } \Omega, \\
\left.y\right|_{\partial \Omega}=0,
\end{array}\right. \\
J(u(\cdot))=\int_{-1}^{1}\left\{y^{2}(x)-u^{2}(x)\right\} d x .
\end{gathered}
$$

Then there is no $\bar{u}(\cdot) \in \mathscr{U}_{\text {ad }}$ such that $J(\bar{u}(\cdot))=\inf _{u(\cdot) \in \mathscr{U}_{\text {sd }}} J(u(\cdot))$. To see this, for $j=1,2, \ldots$, we choose $u_{j}(\cdot)$ as follows:

$$
u_{j}(x)= \begin{cases}1, & |x| \in[k / j,(2 k+1) /(2 j)], k=0,1,2, \ldots, j-1, \\ -1, & \text { otherwise, }\end{cases}
$$

and let $y_{j}(\cdot)$ be the solution of (2.4) corresponding to $u_{j}(\cdot)$. We have $y_{j} \in C^{1, \alpha}[-1,1]$, $\forall \alpha \in(0,1)$, and it is an even function. Therefore $y_{j}^{\prime}(\cdot)$ is odd and $y_{j}^{\prime}(0)=0$. Consequently, by the definition of $u_{j}(\cdot)$, we have

$$
\left|y_{j}^{\prime}(x)\right|=-y_{j}^{\prime}(|x|)=\int_{0}^{|x|} u_{j}(\xi) d \xi \leq \frac{1}{2 j} .
$$

Hence, noting that $y_{j}(-1)=y_{j}(1)=0$, we have $\left|y_{j}(x)\right|=\left|y_{j}(|x|)-y_{j}(1)\right| \leq 1 /(2 j)$. Then

$$
-2 \leq J\left(u_{j}(\cdot)\right)=\int_{-1}^{1}\left\{y_{j}^{2}(x)-u_{j}^{2}(x)\right\} d x \leq \frac{2}{(2 j)^{2}}-2 .
$$




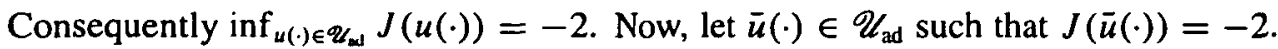
Let $\bar{y}(\cdot)$ be the state corresponding to $\bar{u}(\cdot)$. Then $|\bar{u}(x)|=1$ and $\bar{y}(x)=0$, a.e. on $[-1,1]$. The second relation yields $\bar{u}(x)=0$, a.e. on $[-1,1]$, contradicting the first relation. Thus there is no $\bar{u}(\cdot) \in \mathscr{U}_{\text {ad }}$ such that

$$
J(\bar{u}(\cdot))=-2=\inf _{u(\cdot) \in \mathscr{U}_{\text {sd }}} J(u(\cdot)) .
$$

Now let us introduce a condition of Cesari-type.

DEFINITION 2.1. Let $Y$ be a Banach space and $(Z, d)$ a metric space. Let $\Lambda: Z \rightarrow 2^{Y}$ be a multifunction. We say $\Lambda$ possesses the Cesari property at $z_{0} \in Z$, if

$$
\bigcap_{\delta>0} \overline{\mathrm{co}} \Lambda\left(O_{\delta}\left(z_{0}\right)\right)=\Lambda\left(z_{0}\right)
$$

where $\overline{c o} D$ is the closed convex hull of $D, O_{\delta}\left(z_{0}\right) \equiv\left\{z \in Z \mid d\left(z, z_{0}\right)<\delta\right\}$, and $\Lambda(G) \equiv \bigcup_{z \in G} \Lambda(z)$, for any $G \subseteq Z$. If $\Lambda$ has the Cesari property at every point $z \in Q \subseteq Z$, we simply say that $\Lambda$ has the Cesari property on $Q$.

For any $(x, y) \in \Omega \times \mathbb{R}$, let

$$
\mathscr{E}(x, y) \triangleq\left\{\begin{array}{l|l}
\left(z, z^{0}\right) \in \mathbb{R} \times \mathbb{R} & \begin{array}{l}
z^{0} \geq f^{0}(x, y, u), \\
z=f(x, y, u), \text { for some } u \in U
\end{array}
\end{array} .\right.
$$

To guarantee the existence of a classical optimal control, we usually need to impose the following Cesari-type condition (see $[3,5,11]$ ).

(S5) For almost all $x \in \Omega$, the map $\mathscr{E}(x, \cdot)$ has the Cesari property on $\mathbb{R}$.

By Definition 2.1 , if $\mathscr{E}(x, \cdot)$ has the Cesari property at $y_{0} \in \mathbb{R}$, then $\mathscr{E}\left(x, y_{0}\right)$ is convex and closed. On the other hand, if (S1) and (S3)-(S4) hold, then for almost all $x \in \Omega, \mathscr{E}(x, y)$ is closed for any $y \in \mathbb{R}$. In Example 1 , we can easily verify that (S1)-(S4) hold, while $\mathscr{E}(x, y)=\left\{\left(z, z^{0}\right) \in \mathbb{R} \times \mathbb{R} \mid z^{0} \geq y^{2}-z^{2},-1 \leq z \leq 1\right\}$ is not convex for any $(x, y) \in \Omega \times \mathbb{R}$. Thus (S5) does not hold.

Though (S5) is an important condition to guarantee the existence of classical optimal control, it is not a necessary condition. Here is an example.

EXAMPLE 2. Let $U=[0,1], \Omega=(-1,1)$,

$$
\begin{gathered}
\left\{\begin{array}{c}
-y^{\prime \prime}(x)=u(x), \quad \text { in } \Omega, \\
\left.y\right|_{\partial \Omega}=0,
\end{array}\right. \\
J(u(\cdot))=\int_{-1}^{1}\left\{y^{2}(x)-u^{2}(x)\right\} d x .
\end{gathered}
$$


We mention that Example 2 is very similar to Example 1. In Example 2, (S1)-(S4) still hold but (S5) does not hold. But we will prove in Section 5 that there exists at least one $\bar{u}(\cdot) \in \mathscr{U}_{\text {ad }}$ such that (2.3) holds. Example 2 is a special case of Example 3 in Section 5.

We now recall the notion of relaxed control and state some preliminary results about the space of relaxed controls.

We denote by $\mathscr{M}_{+}^{1}(U)$ the set of all probability measures in $U$, by $\mathscr{R}(\Omega, U)$ the set of all measurable probability measure-valued functions on $\Omega$, that is, $\sigma(\cdot) \in \mathscr{R}(\Omega, \dot{U})$ if and only if $\sigma(x) \in \mathscr{M}_{+}^{1}(U)$, a.e. $x \in \Omega$, and $x \mapsto \int_{U} h(v) \sigma(x)(d v)$ is measurable, $\forall h \in C(U)$, where $C(U)$ denotes the space of continuous functions on $U$. Let $C(U)^{*}$ and $L^{1}(\Omega ; C(U))^{*}$ be the dual spaces of $C(U)$ and $L^{1}(\Omega ; C(U))$ with weak star topology, respectively. We regard $\mathscr{M}_{+}^{1}(U)$ and $\mathscr{R}(\Omega, U)$ as subspaces of $C(U)^{*}$ and $L^{1}(\Omega ; C(U))^{*}$, respectively, by setting

and

$$
\theta(h) \triangleq \int_{U} h(v) \theta(d v), \quad \forall \theta \in \mathscr{M}_{+}^{1}(U), h \in C(U),
$$

$$
\sigma(g) \triangleq \int_{\Omega} d x \int_{U} g(x, v) \sigma(x)(d v), \quad \forall \sigma \in \mathscr{R}(\Omega, U), g \in L^{\prime}(\Omega ; C(U)) .
$$

We see that (2.6) is well-defined by Theorem IV.1.6, (p. 266) in [20]. Thus $\sigma_{k} \rightarrow \sigma$ in $\mathscr{R}(\Omega, U)$ means that

$$
\int_{\Omega} d x \int_{U} h(x, v) \sigma_{k}(x)(d v) \rightarrow \int_{\Omega} d x \int_{U} h(x, v) \sigma(x)(d v), \quad \forall h \in L^{1}(\Omega ; C(U)) .
$$

We now state the optimal relaxed control problem corresponding to Problem (C).

Problem (R). Find a $\bar{\sigma}(\cdot) \in \mathscr{R}(\Omega, U)$ such that

where

$$
J(\bar{\sigma}(\cdot)) \triangleq \inf _{\sigma(\cdot) \in \mathscr{\mathscr { R }}(\Omega, U)} J(\sigma(\cdot)),
$$

$$
J(\sigma(\cdot)) \triangleq \int_{\Omega} d x \int_{U} f^{0}(x, y(x), v) \sigma(x)(d v),
$$

and $y(\cdot)$ is the state corresponding to relaxed control $\sigma(\cdot) \in \mathscr{R}(\Omega, U)$, that is, it is the solution of the following:

$$
\left\{\begin{array}{l}
-\sum_{i, j=1}^{n} \frac{\partial}{\partial x_{i}}\left(a_{i j}(x) \frac{\partial y}{\partial x_{j}}(x)\right)=\int_{U} f(x, y(x), v) \sigma(x)(d v), \quad \text { in } \Omega, \\
\left.y\right|_{\partial \Omega}=0 .
\end{array}\right.
$$


We need to explain the meaning of (2.7) since $f^{0}$ is only supposed to be lower semicontinuous in $(y, v) \in R \times U$. It is not very hard to prove that since $f^{0}$ satisfies (S4), it is the limit of an increasing sequence of functions in $L^{1}(\Omega ; \mathscr{K}(\mathbb{R} \times U)$ ), where $\mathscr{K}(\mathbb{R} \times U)$ denotes the set of all continuous functions in $\mathbb{R} \times U$ with compact supports. Thus we may find $h_{k}(\cdot) \in L^{\prime}(\Omega ; \mathscr{K}(\mathbb{R} \times U)$ ) such that

$$
h_{k}(x, y, v) \uparrow f^{0}(x, y, v), \quad \forall(x, y, v) \in \Omega \times \mathbb{R} \times U .
$$

Consequently, we-can define $J(\sigma(\cdot))$ by identifying the right-hand side of $(2.7)$ with the following limit:

$$
\lim _{k \rightarrow+\infty} \int_{\Omega} d x \int_{U} h_{k}(x, y(x), v) \sigma(x)(d v) .
$$

We mention that $\mathscr{U}_{\text {ad }}$ can be imbedded into $\mathscr{R}(\Omega, U)$ by identifying each $u(\cdot) \in \mathscr{U}_{\text {ad }}$ with the Dirac measure-valued function $\delta_{u(\cdot)} \in \mathscr{R}(\Omega, U)$. Moreover, $J\left(\delta_{u(\cdot)}\right)$ defined by (2.7) coincides with $J(u(\cdot))$ defined by (2.2). Thus the notation $J(\sigma(\cdot))$ does not cause any confusion. On the other hand, it is easy to see that if $\sigma(\cdot) \in \mathscr{R}(\Omega, U)$ and there exists a $u: \Omega \rightarrow U$ such that $\sigma(x)=\delta_{u(x)}$, a.e. $x \in \Omega$, then $u(\cdot)$ must be measurable, that is, $u(\cdot) \in \mathscr{U}_{\text {ad }}$. Thus, if Problem (R) has an optimal relaxed control $\bar{\sigma}(\cdot) \in \mathscr{R}(\Omega, U)$ such that supp $\bar{\sigma}(x)$ is a singleton of $U$ for almost all $x \in \Omega$, then Problem (C) admits at least one classical optimal control.

The following lemma is crucial in deriving the existence of optimal relaxed controls.

LEMMA 2.2. Suppose $U$ is a compact metric space. Then $\mathscr{R}(\Omega, U)$ is convex and sequentially compact.

For a proof of the above lemma, see Warga [20, Theorem IV.2.1, p. 272].

\section{Existence of an optimal relaxed control}

We begin with a preliminary lemma which shows that (2.8) is well-posed.

LEMMA 3.1. Let (S1)-(S3) hold. Then for any $\sigma(\cdot) \in \mathscr{R}(\Omega, U)$, (2.8) admits a unique weak solution $y(\cdot) \in W_{0}^{1, p}(\Omega) \cap L^{\infty}(\Omega)$, for any $p \in[1,+\infty)$. Furthermore, there exist constants $C_{p}>0$ and $M>0$ independent of $\sigma(\cdot) \in \mathscr{R}(\Omega, U)$, such that

$$
\left\{\begin{array}{rl}
\|y(\cdot)\|_{w_{0}^{1, p}(\Omega)} & \leq C_{p}, \quad \\
\|y(\cdot)\|_{C(\Omega)} & \leq M,
\end{array} \quad \forall \sigma \in \mathscr{R}(\Omega, U) .\right.
$$

The above lemma is basic. We omit the proof since it is similar to that for (2.2), see, for example, [11, Chapter 2, Theorem 6.11 and Chapter 3, Proposition 6.3].

We now state the existence theorem of optimal relaxed controls. 
THEOREM 3.2. Let (S1)-(S4) hold. Then Problem (R) admits at least one solution.

PROOF. We give only a sketch of the proof since it is quite standard.

By (S4) and Lemma 3.1, we have a sequence $\sigma_{k}(\cdot) \in \mathscr{R}(\Omega, U)$, such that

$$
J\left(\sigma_{k}(\cdot)\right) \rightarrow \bar{J} \equiv \inf _{\sigma(\cdot) \in \mathscr{R}(\Omega, U)} J(\sigma(\cdot)) .
$$

Let $y_{k}(\cdot)$ be the state corresponding to $\sigma_{k}(\cdot)$. Then, choosing a subsequence if necessary, we can suppose that $\sigma_{k}(\cdot) \rightarrow \bar{\sigma}(\cdot)$ in $\mathscr{R}(\Omega, U), y_{k}(\cdot) \rightarrow \bar{y}(\cdot)$ weakly in $W_{0}^{1, p}(\Omega)$, uniformly in $C(\bar{\Omega})$, by Lemmas 2.2 and 3.1, and the Sobolev imbedding theorem, where we set $p>n$. Thus it is not very hard to check that $\bar{y}(\cdot)$ is the state corresponding to $\bar{\sigma}(\cdot)$. By Theorem IV.2.9 in [20], we have, $\forall h \in L^{1}(\Omega ; \mathscr{K}(\mathbb{R} \times U))$,

$$
\lim _{k \rightarrow+\infty} \int_{\Omega} d x \int_{U} h\left(x, y_{k}(x), v\right) \sigma_{k}(x)(d v)=\int_{\Omega} d x \int_{U} h(x, \bar{y}(x), v) \bar{\sigma}(x)(d v) .
$$

Let $h_{j}$ be an increasing sequence satisfying (2.9). Then

$$
\begin{aligned}
J(\bar{\sigma}(\cdot)) & =\int_{\Omega} d x \int_{U} f^{0}(x, \bar{y}(x), v) \bar{\sigma}(x)(d v) \\
& =\lim _{j \rightarrow+\infty} \int_{\Omega} d x \int_{U} h_{j}(x, \bar{y}(x), v) \bar{\sigma}(x)(d v) \\
& =\lim _{j \rightarrow+\infty} \lim _{k \rightarrow+\infty} \int_{\Omega} d x \int_{U} h_{j}\left(x, y_{k}(x), v\right) \sigma_{k}(x)(d v) \\
& \leq \lim _{j \rightarrow+\infty} \lim _{k \rightarrow+\infty} \int_{\Omega} d x \int_{U} f^{0}\left(x, y_{k}(x), v\right) \sigma_{k}(x)(d v) \\
& =\lim _{k \rightarrow+\infty} \int_{\Omega} d x \int_{U} f^{0}\left(x, y_{k}(x), v\right) \sigma_{k}(x)(d v) \\
& =\bar{J} .
\end{aligned}
$$

Therefore $\bar{\sigma}(\cdot)$ is an optimal relaxed control to Problem (R).

We now recall the definition of $\mathscr{E}(x, y)$ (see (2.5)). Under assumptions (S1) and (S3)-(S4), $\mathscr{E}(x, \bar{y}(x))$ is closed for almost all $x \in \Omega$. If it is also convex for almost all $x \in \Omega$, then, since

$$
\begin{gathered}
\left(\int_{U} f(x, \bar{y}(x), v) \bar{\sigma}(x)(d v), \int_{U} f^{0}(x, \bar{y}(x), v) \bar{\sigma}(x)(d v)\right) \\
\in \overline{\mathrm{co}} \mathscr{E}(x, \bar{y}(x))=\mathscr{E}(x, \bar{y}(x)), \quad \text { a.e. } x \in \Omega,
\end{gathered}
$$

we have $\tilde{u}: \Omega \rightarrow U$, not necessarily measurable, such that for almost all $x \in \Omega$,

$$
\left\{\begin{array}{l}
\int_{U} f(x, \bar{y}(x), v) \bar{\sigma}(x)(d v)=f(x, \bar{y}(x), \tilde{u}(x)), \\
\int_{U} f^{0}(x, \bar{y}(x), v) \bar{\sigma}(x)(d v) \geq f^{0}(x, \bar{y}(x), \tilde{u}(x)) .
\end{array}\right.
$$


Thus, by Filippov's lemma (see [7] and [11, Corollary 2.26, Chapter 3]), which is usually called the "implicit measurable function theorem", there exists a measurable $\bar{u}(\cdot) \in \mathscr{U}_{\text {ad }}$ such that

$$
\left\{\begin{array}{l}
\int_{U} f(x, \bar{y}(x), v) \bar{\sigma}(x)(d v)=f(x, \bar{y}(x), \bar{u}(x)), \\
\int_{U} f^{0}(x, \bar{y}(x), v) \bar{\sigma}(x)(d v) \geq f^{0}(x, \bar{y}(x), \bar{u}(x)) .
\end{array}\right.
$$

Replacing $\bar{\sigma}(\cdot)$ by $\bar{u}(\cdot)$ (that is, $\delta_{\bar{u}(\cdot)}$ ), the value of the right-hand side of the first relation in (2.8) remains unchanged. Thus $\bar{y}(\cdot)$ is also the state corresponding to $\bar{u}(\cdot)$. Consequently, by (3.2), $J(\bar{u}(\cdot)) \leq J(\bar{\sigma}(\cdot))$. Therefore $\bar{u}(\cdot)$ must be a classical optimal control to Problem (C).

The above tells us that if we have

(S5)' For almost all $x \in \Omega, \mathscr{E}(x, y)$ is convex for any $y \in \mathbb{R}$, then Problem (C) admits an optimal control.

In fact, under assumptions (S1)-(S4), (S5) holds if and only if (S5)' holds (see LiYong [11, Proposition 4.3, p. 107]). Thus we have obtained a proof for the existence of optimal controls to Problem (C) under (S1)-(S5). We would like to mention that such a proof is essentially the same as that given in [11, pp. 127-128].

On the other hand, replacing $U$ and $f(x, y(x), u(x))$ by $\mathscr{M}_{+}^{1}(U)$ and

$$
f(x, y(x), \sigma(x)) \equiv \int_{U} f(x, y(x), v) \sigma(x)(d v),
$$

respectively, and so on, the relaxed control system (2.7)-(2.8) is a special case of control system (2.1)-(2.2). Thus Theorem 3.2 is in fact a special case of [11, Theorem 6.4]. The proof of Theorem 3.2 is essentially a procedure of verifying that the relaxed control system (2.7)-(2.8) satisfies the assumptions (especially the lower semicontinuity) needed for [11, Theorem 6.4]. For the fact of $\mathscr{M}_{+}^{1}(U)$ being a compact metric space, see Warga [20, Theorem IV.1.4, p. 625].

\section{Maximum principle for optimal relaxed controls}

To derive a maximum principle for optimal relaxed controls, we make some further assumptions.

(S3)' $f: \Omega \times \mathbb{R} \times U \rightarrow \mathbb{R}$ has the following properties: $f(\cdot, y, u)$ is measurable on $\Omega$, and $f(x, \cdot, u)$ is in $C^{1}(\mathbb{R})$ with $f(x, \cdot, \cdot)$ and $f_{y}(x, \cdot, \cdot)$ continuous on $\mathbb{B} \times U$. Moreover,

$$
f_{y}(x, y, u) \leq 0, \quad \forall(x, y, u) \in \Omega \times \mathbb{R} \times U,
$$


and for any $R>0$, there exists an $M_{R}>0$ such that

$$
|f(x, y, u)|+\left|f_{y}(x, y, u)\right| \leq M_{R}, \quad \forall(x, u) \in \Omega \times U,|y| \leq R .
$$

(S4) The function $f^{0}: \Omega \times \mathbb{R} \times U \rightarrow \mathbb{R}$ satisfies (S3)' except for (4.1).

The maximum principle of optimal relaxed controls can be established essentially the same way as that for classical control problems. Thus we omit the details and only state the result.

THEOREM 4.1. Let (S1)-(S2) and (S3)'-(S4)' hold. Let $(\bar{y}(\cdot), \bar{\sigma}(\cdot))$ be an optimal relaxed pair to Problem (R). Then there exists a $\bar{\psi}(\cdot) \in W_{0}^{1, p}(\Omega)(\forall p \in[1,+\infty))$ such that

$$
\begin{aligned}
& \left\{\begin{array}{l}
-\sum_{\substack{i, j=\left.1 \\
\bar{y}\right|_{\partial \Omega}=0,}}^{n} \frac{\partial}{\partial x_{i}}\left(a_{i j}(x) \frac{\partial \bar{y}}{\partial x_{j}}(x)\right)=\int_{U} f(x, \bar{y}(x), v) \bar{\sigma}(x)(d v), \quad \text { in } \Omega,
\end{array}\right. \\
& \left\{\begin{aligned}
-\sum_{i, j=1}^{n} \frac{\partial}{\partial x_{i}}\left(a_{i j}(x) \frac{\partial \bar{\psi}}{\partial x_{j}}(x)\right)= & \int_{U} f_{y}(x, \bar{y}(x), v) \bar{\sigma}(x)(d v) \bar{\psi}(x) \\
& -\int_{U} f_{y}^{0}(x, \bar{y}(x), v) \bar{\sigma}(x)(d v), \quad \text { in } \Omega, \\
\left.\bar{\psi}\right|_{\partial \Omega}=0, &
\end{aligned}\right.
\end{aligned}
$$

and, $\forall \sigma \in \mathscr{R}(\Omega, U)$,

$$
\int_{\Omega} d x \int_{U}\left(f(x, \bar{y}(x), v) \bar{\psi}(x)-f^{0}(x, \bar{y}(x), v)\right)(\sigma(x)-\bar{\sigma}(x))(d v) \leq 0 .
$$

We call (4.3) the adjoint equation of the variational system along the optimal pair. In the current case, the maximum condition takes the variational inequality form (4.4) (comparing with that found in [11]). Next, we denote

$$
H(x, y, w, \psi) \equiv f(x, y, w) \psi-f^{0}(x, y, w)
$$

and

$$
U_{x} \equiv\left\{w \in U \mid H(x, \bar{y}(x), w, \bar{\psi}(x))=\max _{v \in U} H(x, \bar{y}(x), v, \bar{\psi}(x))\right\} .
$$

Then it is easy to see that (4.4) is equivalent to

$$
\operatorname{supp} \bar{\sigma}(x) \subseteq U_{x}, \quad \text { a.e. } x \in \Omega .
$$

Proposition 4.2. Under the assumptions of Theorem 4.1, let $(\bar{y}(\cdot), \bar{\sigma}(\cdot))$ be an optimal relaxed pair to Problem (R) and $U_{x}$ be defined by (4.5). If for almost all $x \in \Omega$, either $U_{x}$ is a singleton of $U$ or $\mathscr{E}(x, \bar{y}(x))$ is convex, then Problem (C) admits at least one classical optimal control. 
Proof. Let $\Omega_{0} \triangleq\left\{x \in \Omega \mid U_{x}\right.$ is a singleton $\}$. Then for any $x \in \Omega_{0}$, we have $\bar{u}(x) \in U$ such that $\bar{\sigma}(x)=\delta_{\bar{u}(x)}$. Therefore

$$
\left\{\begin{array}{l}
\int_{U} f(x, \bar{y}(x), v) \bar{\sigma}(x)(d v)=f(x, \bar{y}(x), \bar{u}(x)), \\
\int_{U} f^{0}(x, \bar{y}(x), v) \bar{\sigma}(x)(d v)=f^{0}(x, \bar{y}(x), \bar{u}(x)),
\end{array} \forall x \in \Omega_{0} .\right.
$$

On the other hand, for almost all $x \in \Omega \backslash \Omega_{0}, \mathscr{E}(x, \bar{y}(x))$ is convex. By (S1) and (S3)-(S4), $\mathscr{E}(x, \bar{y}(x))$ is closed for almost all $x \in \Omega$. Thus we have $\bar{u}(x) \in U$ such that (3.2) holds for almost all $x \in \Omega \backslash \Omega_{0}$. Combining the above with (4.7), we see that there exists $\bar{u}: \Omega \rightarrow U$, not necessarily measurable, such that (3.2) holds for almost all $x \in \Omega$. Then, by Filippov's lemma, we can change the definition of $\bar{u}(\cdot)$ such that $\bar{u}(\cdot) \in \mathscr{U}_{\mathrm{ad}}$ and (3.2) still holds for almost all $x \in \Omega$. Obviously, $\bar{y}(\cdot)$ is the state corresponding to $\bar{u}(\cdot)$ satisfying $(2.1)$ and $J(\bar{u}(\cdot)) \leq J(\bar{\sigma}(\cdot))$. Therefore $\bar{u}(\cdot)$ must be an optimal classical control to Problem (C).

PROPOSITION 4.3. Under the assumptions of Theorem 4.1 , let $(\bar{y}(\cdot), \bar{\sigma}(\cdot))$ be an optimal relaxed pair to Problem (R). Suppose for almost all $x \in \Omega$,

$$
f(x, \bar{y}(x), v)=f(x, \bar{y}(x), w), \quad \forall v, w \in \operatorname{supp} \bar{\sigma}(x) .
$$

Then Problem (C) admits at least one classical optimal control.

Proof. By (4.6), (4.8), and the definition of $U_{x}$, we get for almost all $x \in \Omega$,

$$
f^{0}(x, \bar{y}(x), v)=f^{0}(x, \bar{y}(x), w), \quad \forall v, w \in \operatorname{supp} \bar{\sigma}(x) .
$$

Thus let $\overline{\boldsymbol{u}}: \Omega \rightarrow U$, not necessarily measurable, such that $\bar{u}(x) \in \operatorname{supp} \bar{\sigma}(x)$. We have that (4.7) holds for almost all $x \in \Omega$. Therefore, by Filippov's lemma, we can find a $\bar{u}(\cdot) \in \mathscr{U}_{\text {ad }}$ such that (4.7) holds for almost all $x \in \Omega$. As we have seen in the proof of Proposition 4.2, such a $\bar{u}(\cdot)$ must be an optimal classical control to Problem (C).

\section{Existence of a classical optimal control}

In this section, we will state and prove our main theorem. We consider the following system:

$$
\left\{\begin{array}{l}
-\sum_{i, j=1}^{n} \frac{\partial}{\partial x_{i}}\left(a_{i j}(x) \frac{\partial y}{\partial x_{j}}(x)\right)=f(x, y(x))+g(u(x)), \quad \text { in } \Omega, \\
\left.y\right|_{\partial \Omega}=0 .
\end{array}\right.
$$


Let the cost functional take the form

$$
J(u(\cdot))=\int_{\Omega}\left\{f^{0}(x, y(x))+h(u(x))\right\} d x,
$$

where $y(\cdot)$ is the state corresponding to control $u(\cdot)$ satisfying (5.1).

We impose the following assumptions.

(P1) (S1) holds.

(P2) In addition to (S2), $a_{i j}(\cdot) \in C^{1}(\Omega)$.

(P3) $g \in C(U)$. The function $f: \Omega \times \mathbb{R} \rightarrow \mathbb{R}$ satisfies (S3)'.

(P4) $h \in C(U)$. The function $f^{0}: \Omega \times \mathbb{R} \rightarrow \mathbb{R}$ satisfies (S4)'.

Denote $a=\min _{u \in U} g(u)$ and $b=\max _{u \in U} g(u)$. Let $y_{\min }(\cdot)$ and $y_{\max }(\cdot)$ be the solution of (5.1) corresponding to $g(u(x)) \equiv a$ and $g(u(x)) \equiv b$, respectively. Before making further assumptions, let us introduce the following lemma.

LEMMA 5.1. Suppose that $U$ is a compact metric space and $g, h \in C(U)$. For any $\beta \in \mathbb{R}$, denote

$$
\begin{aligned}
& E_{\beta} \triangleq\left\{w \in U \mid \beta g(w)-h(w)=\max _{v \in U}[\beta g(v)-h(v)]\right\}, \\
& a_{\beta}=\min _{w \in E_{\beta}} g(w), \quad b_{\beta}=\max _{w \in E_{\beta}} g(w), \quad F \triangleq\left\{\beta \in \mathbb{R} \mid a_{\beta}<b_{\beta}\right\} .
\end{aligned}
$$

Then $F$ is at most countable.

PROOF. Since $g(\cdot)$ and $h(\cdot)$ are continuous in $U$ and $U$ is compact, $E_{\beta}, a_{\beta}$ and $b_{\beta}$ are well-defined for any $\beta \in \mathbb{R}$.

Suppose $\beta \in F, \tilde{\beta} \in \mathbb{R}$ and $\tilde{w} \in E_{\tilde{\beta}}$ such that

$$
a_{\beta}<g(\tilde{w})<b_{\beta} .
$$

We will prove that $\tilde{\beta}=\beta$.

By the definition of $E_{\beta}, a_{\beta}$ and $b_{\beta}$, we have $w_{1}, w_{2} \in E_{\beta}$ such that

$$
a_{\beta}=g\left(w_{1}\right), \quad b_{\beta}=g\left(w_{2}\right),
$$

and $\beta g\left(w_{i}\right)-h\left(w_{i}\right) \geq \beta g(\tilde{w})-h(\tilde{w}), i=1,2$. Similarly, by the definition of $E_{\tilde{\beta}}$, we have $\tilde{\beta} g(\tilde{w})-h(\tilde{w}) \geq \tilde{\beta} g\left(w_{i}\right)-h\left(w_{i}\right), i=1,2$. Thus we have

$$
(\beta-\tilde{\beta})\left(g\left(w_{i}\right)-g(\tilde{w})\right) \geq 0, \quad i=1,2 .
$$

Therefore, by (5.3)-(5.5), we get $\tilde{\beta}=\beta$.

The above implies that if $\beta, \tilde{\beta} \in F$ and $\beta \neq \tilde{\beta}$, then $\left(a_{\beta}, b_{\beta}\right) \cap\left(a_{\tilde{\beta}}, b_{\tilde{\beta}}\right)=\emptyset$. Thus the number of nonempty $\left(a_{\beta}, b_{\beta}\right)$ 's is at most countable, that is, $F$ is at most countable. 
Now we suppose that the following additional assumption also holds:

(P5) Let $a, b$ and $F$ be defined as above. For any $\beta \in F$, there exist a subset $\Omega_{\beta}$ of $\Omega$ and two sequences of functions $Y_{\beta, k}(\cdot)$ and $Z_{\beta, k}(\cdot)$ in $W_{\text {loc }}^{2,1}(\Omega)$ such that

$$
\left|\Omega \backslash \Omega_{\beta}\right| \equiv \text { the Lebesgue measure of } \Omega \backslash \Omega_{\beta}=0
$$

and

$$
\begin{aligned}
& \left\{(x, y) \in \Omega_{\beta} \times \mathbb{B} \mid f_{y}^{0}(x, y)=\beta f_{y}(x, y)\right\} \\
& \subseteq\left\{(x, y) \in \Omega_{\beta} \times \mathbb{R} \mid y=Y_{\beta, k}(x), \text { for some } k=1,2, \ldots\right\} \\
& \cup\left\{(x, y) \in \Omega_{\beta} \times \mathbb{R} \mid y=Z_{\beta, k}(x), \text { for some } k=1,2, \ldots\right\} \\
& \cup\left\{(x, y) \in \Omega_{\beta} \times \mathbb{B} \mid y>y_{\max }(x) \text { or } y<y_{\min }(x)\right\},
\end{aligned}
$$

while, a.e. on $\Omega, \forall k=1,2, \ldots$,

$$
\begin{aligned}
& -\sum_{i, j=1}^{n} \frac{\partial}{\partial x_{i}}\left(a_{i j}(x) \frac{\partial Y_{\beta, k}}{\partial x_{j}}(x)\right) \leq f\left(x, Y_{\beta, k}(x)\right)+a, \\
& -\sum_{i, j=1}^{n} \frac{\partial}{\partial x_{i}}\left(a_{i j}(x) \frac{\partial Z_{\beta, k}}{\partial x_{j}}(x)\right) \geq f\left(x, Z_{\beta, k}(x)\right)+b .
\end{aligned}
$$

For our problem, we have

$$
\mathscr{E}(x, y)=\left\{\begin{array}{l|l}
\left(z, z^{0}\right) \in \mathbb{R} \times \mathbb{R} & \begin{array}{l}
z^{0} \geq f^{0}(x, y)+h(u), \\
z=f(x, y)+g(u), \text { for some } u \in U
\end{array}
\end{array} .\right.
$$

Clearly $\mathscr{E}(x, y)$ is not convex in general. Therefore, for such systems, the Cesari-type condition (S5) does not hold in general.

We now state our main theorem.

THEOREM 5.2. Let (P1)-(P5) hold. Then Problem (C) corresponding to system (5.1)-(5.2) admits at least one optimal control.

In applications, we may replace (P5) by the following stronger condition:

(P5)' For any $\beta \in \mathbb{R}$, there exist $\Omega_{\beta} \subseteq \Omega$, and two sequences of functions $Y_{\beta, k}(\cdot)$, $Z_{\beta, k}(\cdot) \in W_{\text {loc }}^{2,1}(\Omega)$ such that (5.6)-(5.8) hold.

The advantage of (P5)' is that it is independent of $g(\cdot)$ and $h(\cdot)$, except for the upper and lower bounds of $g(\cdot)$.

Assumption (P5) looks very technical. But we will see that many systems satisfy such a condition.

EXAMPLE 3. Consider the system (5.1)-(5.2). Let

$$
f(x, y)=r(y), \quad f^{0}(x, y)=r^{0}(y), \quad \forall(x, y) \in \Omega \times \mathbb{R} .
$$


Let (P1)-(P4) hold. Moreover, suppose $\left\{y \mid\left(r^{0}\right)^{\prime}(y)=\beta r^{\prime}(y)\right\}$ is at most countable and $\inf _{y \in \mathbb{R}} r(y)+\min _{v \in U} g(v) \geq 0$. Then (P5)' (and consequently (P5)) follows from the following relation:

$$
\begin{aligned}
& \left\{(x, y) \in \Omega \times \mathbb{R} \mid f_{y}^{0}(x, y)=\beta f_{y}(x, y)\right\} \\
& =\left\{(x, y) \in \Omega \times \mathbb{R} \mid y=C_{\beta, k}, \text { for some } k=1,2, \ldots\right\},
\end{aligned}
$$

where $C_{\beta, k} \in\left\{y \mid\left(r^{0}\right)^{\prime}(y)=\beta r^{\prime}(y)\right\}$. Comparing the above with (5.6)-(5.9), we see that it suffices to set $\Omega_{\beta}=\Omega$ and $Y_{\beta, k}(\cdot) \equiv C_{\beta, k}$, while $Z_{\beta, k}(\cdot)$ is not necessary.

By Theorem 5.2, for such a system, there exists an optimal classical control to Problem (C).

Let us give some special cases of the above example.

EXAMPLE 4. In Example 3, let $g \geq 0$. Then it is easy to see that we can choose $r(y)=0$ (or $e^{-y}$, or $-\arctan y+\pi / 2$, etc.) and $r^{0}(y)=$ a polynomial of $y$ (or a polynomial of $e^{y}$, or a trigonometric polynomial of $y$, or $\ln \left(1+y^{2}\right)$, or $\sqrt{1+y^{2}}, e t c$.).

REMARK. The trivial case $r^{0} \equiv 0$ is an exception. In this case, (P5) does not hold because $\left\{y \in \mathbb{R} \mid\left(r^{0}\right)^{\prime}(y)=\beta r^{\prime}(y)\right\}=\mathbb{R}$ when $\beta=0$.

Clearly, the system described in Example 3 does not satisfy the Cesari-type condition (S5) in general. Example 2 is just a special case of Example 3, which does not satisfy (S5). The following two examples also do not satisfy condition (S5). It is interesting to compare them with Example 1.

EXAMPLE 5. Let $\Omega$ satisfy (P1), $U=[-1,1]$. Consider the following system:

$$
\left\{\begin{array}{l}
-\Delta y(x)=-y(x)+\cos y(x)+u(x), \quad \text { in } \Omega, \\
\left.y\right|_{\partial \Omega}=0 .
\end{array}\right.
$$

The cost functional is $J(u(\cdot))=\int_{\Omega}\left\{y^{2}(x)-u^{2}(x)\right\} d x$. It is easy to verify that $a_{\beta}<b_{\beta} \Longleftrightarrow \beta=0$, that is, $F=\{0\}$. Let $Y(x) \equiv 0$. Then

$$
-\triangle Y(x) \leq-Y(x)+\cos Y(x)+\min _{v \in U} v, \quad \text { in } \Omega .
$$

Thus we can see that (P5) holds (here (P5)' does not hold). Since (P1)-(P4) hold obviously, by Theorem 5.2 , there exists a measurable $\bar{u}(\cdot): \Omega \rightarrow U$ such that

$$
J(\bar{u}(\cdot))=\inf _{u(\cdot) \in \mathscr{U} \mathscr{U}_{\mathrm{d}}} J(u(\cdot))
$$


EXAMPLE 6. Let $\Omega=(-1,1), U=[-1,1]$,

$$
\begin{aligned}
& \left\{\begin{array}{l}
-y^{\prime \prime}(x)=u(x), \quad \text { in } \Omega, \\
\left.y\right|_{\partial \Omega}=0,
\end{array}\right. \\
J(u(\cdot))= & \int_{-1}^{1}\left\{|y(x)-M \operatorname{sgn}(x)|^{2}-u^{2}(x)\right\} d x,
\end{aligned}
$$

where $M$ is sufficiently large. In fact, it suffices to take $M>1 / 2$. In this example, we can see that (5.7) holds since

$$
\begin{aligned}
& \{(x, y) \in \Omega \times \mathbb{R} \mid y-M \operatorname{sgn}(x)=0\} \\
& \quad \subseteq\left\{(x, y) \in \Omega \times \mathbb{R} \mid y>y_{\max }(x) \text { or } y<y_{\min }(x)\right\}
\end{aligned}
$$

On the other hand, (P1)-(P4) obviously hold. Thus, by Theorem 5.2, we have a $\bar{u}(\cdot) \in \mathscr{U}_{\text {ad }}$ such that

$$
J(\bar{u}(\cdot))=\inf _{u(\cdot) \in \mathscr{Q}_{\mathrm{od}}} J(u(\cdot))
$$

Now we turn to proving Theorem 5.2. Let us first introduce the following lemma.

LEMMA 5.3. Let $C$ be a constant. If $\varphi \in W^{m, p}(\Omega), p \geq 1, m \geq 1$, then

$$
\partial^{\rho} \varphi(x)=0, \text { a.e. }\{\varphi=C\}, \forall 1 \leq|\rho| \leq m,
$$

where $\rho=\left(\rho_{1}, \cdots, \rho_{n}\right)$ is an $n$-tuple of nonnegative integers $\rho_{i},|\rho|=\sum_{i=1}^{n} \rho_{i}$.

In the case where $m=1$, the above result can be found in Morrey [14, p. 69]. See also Kinderlehrer and Stampacchia [10, Chapter 2]. The remaining cases can be obtained easily by induction.

PROOF OF THEOREM 5.2. By Theorem 4.1, Problem (R) admits an optimal relaxed pair $(\bar{y}(\cdot), \bar{\sigma}(\cdot)) \in W_{0}^{1, p}(\Omega) \times \mathscr{R}(\Omega, U)(1 \leq p<+\infty)$. Moreover, there exists a $\bar{\psi}(\cdot) \in W_{0}^{1, p}(\Omega)$ such that

$$
\begin{aligned}
& \left\{\begin{array}{l}
-\sum_{i, j=1}^{n} \frac{\partial}{\partial x_{i}}\left(a_{i j}(x) \frac{\partial \bar{y}}{\partial x_{j}}(x)\right)=f(x, \bar{y}(x))+\int_{U} g(v) \bar{\sigma}(x)(d v), \quad \text { in } \Omega, \\
\left.\bar{y}\right|_{\partial \Omega}=0
\end{array}\right. \\
& \left\{\begin{array}{l}
-\sum_{i, j=1}^{n} \frac{\partial}{\partial x_{i}}\left(a_{i j}(x) \frac{\partial \bar{\psi}}{\partial x_{j}}(x)\right)=f_{y}(x, \bar{y}(x)) \bar{\psi}(x)-f_{y}^{0}(x, \bar{y}(x)), \quad \text { in } \Omega, \\
\left.\bar{\psi}\right|_{\partial \Omega}=0
\end{array}\right.
\end{aligned}
$$


and

$$
\operatorname{supp} \bar{\sigma}(x) \subseteq U_{x}, \quad \text { a.e. } x \in \Omega,
$$

where $U_{x}=\left\{w \in U \mid g(w) \bar{\psi}(x)-h(w)=\max _{v \in U}(g(v) \bar{\psi}(x)-h(v))\right\}, \forall x \in \Omega$. By Lemma 3.1 and (P3), we see that $|f(x, \bar{y}(x))| \leq C$, a.e. $x \in \Omega$, for some constant $C>0$. Moreover, $\left|\int_{U} g(v) \bar{\sigma}(x)(d v)\right| \leq \max _{v \in U}|g(v)|$, a.e. $x \in \Omega$. Consequently, since $a_{i j}(\cdot) \in C^{1}(\Omega)$, by an $L^{p}$-estimate for the elliptic equations, $\bar{y}(\cdot) \in W_{\mathrm{loc}}^{2, p}(\Omega)$ for any $p \in[1,+\infty)$. Similarly, $\bar{\psi}(\cdot) \in W_{\mathrm{loc}}^{2, p}(\Omega)$ for any $p \in[1,+\infty)$.

Denote $\mathscr{Q} \equiv\{x \in \Omega \mid \bar{\psi}(x) \in F\}$. By Lemma 5.1 , we have $\beta_{1}, \beta_{2}, \ldots, \beta_{k}, \ldots$, such that $\mathscr{Q} \subseteq \bigcup_{k}\left\{x \in \Omega \mid \bar{\psi}(x)=\beta_{k}\right\}$. Denote $\mathscr{Q}_{k} \equiv\left\{x \in \Omega \mid \bar{\psi}(x)=\beta_{k}\right\} \cap \Omega_{\beta_{k}}$. Then $\left|\bigcup_{k} \mathscr{Q}_{k} \backslash \mathscr{Q}\right|=0$. Since $a_{i j}(\cdot) \in C^{\prime}(\Omega)$ and $\bar{\psi}(\cdot) \in W_{\text {loc }}^{2, p}(\Omega)$, we get

$$
\sum_{j=1}^{n} a_{i j}(\cdot) \frac{\partial \bar{\psi}}{\partial x_{j}}(\cdot) \in W_{10 c}^{1, p}(\Omega), \quad \forall i=1,2, \ldots, n,
$$

and by Lemma 5.3,

$$
\sum_{j=1}^{n} a_{i j}(x) \frac{\partial \bar{\psi}}{\partial x_{j}}(x)=0, \quad \text { a.e. } x \in \mathscr{Q}_{k}, \forall i=1,2, \ldots, n ; k=1,2, \ldots
$$

Therefore

$$
-\sum_{i, j=1}^{n} \frac{\partial}{\partial x_{i}}\left(a_{i j}(x) \frac{\partial \bar{\psi}}{\partial x_{j}}(x)\right)=0, \quad \text { a.e. } x \in \mathscr{Q}_{k}, \forall k=1,2, \ldots
$$

That is, $f_{y}(x, \bar{y}(x)) \beta_{k}-f_{y}^{0}(x, \bar{y}(x))=0$, a.e. $x \in \mathscr{Q}_{k}, \forall k=1,2, \ldots$ Therefore, it follows from (P5) that

$$
\begin{aligned}
\mathscr{Q}_{k} \subseteq & \left(\bigcup_{l}\left\{x \in \Omega_{\beta_{k}} \mid \bar{y}(x)=Y_{\beta_{k}, l}(x)\right\}\right) \bigcup\left(\bigcup_{l}\left\{x \in \Omega_{\beta_{k}} \mid \bar{y}(x)=Z_{\beta_{k}, l}(x)\right\}\right) \\
& \bigcup\left\{x \in \Omega_{\beta_{k}} \mid \bar{y}(x)<y_{\min }(x) \text { or } \bar{y}(x)>y_{\max }(x)\right\} \\
\equiv & \left(\bigcup_{l} F_{k, l}^{(1)}\right) \bigcup\left(\bigcup_{l} F_{k, l}^{(2)}\right) \bigcup F_{k}^{(3)} .
\end{aligned}
$$

Since

$$
\int_{U} g(v) \bar{\sigma}(x)(d v) \in[a, b]
$$

hence $y_{\min }(x) \leq \bar{y}(x) \leq y_{\max }(x)$, a.e. on $\Omega$. Thus $\left|F_{k}^{(3)}\right|=0, \forall k=1,2, \ldots$ 
On the other hand, for each $l$, since $\bar{y}(\cdot) \in W^{2, p}(\Omega)$ and $Y_{\beta_{k}, l} \in W_{\mathrm{loc}}^{2,1}(\Omega)$, similar to $(5.12)$, we have

$$
\begin{aligned}
-\sum_{i, j=1}^{n} \frac{\partial}{\partial x_{i}}\left(a_{i j}(x) \frac{\partial \bar{y}}{\partial x_{j}}(x)\right) & =-\sum_{i, j=1}^{n} \frac{\partial}{\partial x_{i}}\left(a_{i j}(x) \frac{\partial Y_{\beta_{k}, l}}{\partial x_{j}}(x)\right) \\
& \leq f\left(x, Y_{\beta_{k}, l}(x)\right)+a=f(x, \bar{y}(x))+a,
\end{aligned}
$$

a.e. $x \in F_{k, l}^{(1)} \equiv\left\{x \in \Omega_{\beta_{k}} \mid \bar{y}(x)=Y_{\beta_{k}, l}(x)\right\}$. Thus, by (5.10), $\int_{U} g(v) \bar{\sigma}(x)(d v) \leq a$, a.e. $x \in F_{k, l}^{(i)}$. Therefore, by the definition of $a$, we have

Similarly,

$$
\operatorname{supp} \bar{\sigma}(x) \subseteq\{v \in U \mid g(v)=a\}, \quad \text { a.e. } x \in F_{k, l}^{(1)}
$$

$$
\operatorname{supp} \bar{\sigma}(x) \subseteq\{v \in U \mid g(v)=b\}, \quad \text { a.e. } x \in F_{k, l}^{(2)} .
$$

Thus we see that for almost all $x \in \mathscr{Q}$,

$$
g(v)=g(w), \quad \forall v, w \in \operatorname{supp} \bar{\sigma}(x) .
$$

On the other hand, $\forall x \in \Omega \backslash \mathscr{Q}, \bar{\psi}(x) \notin F$, that is,

$$
g(v)=g(w), \quad \forall v, w \in U_{x} .
$$

Combining (5.14)-(5.15) with (5.11), we see that (5.14) holds for almost all $x \in \Omega$. By Proposition 4.3, Problem (C) has at least one classical optimal control. We complete the proof.

\section{Acknowledgements}

This work was supported in part by the Science Foundation of the Education Ministry of China. It was written under the guidance of Professor Jiongmin Yong. Many valuable suggestions were offered by Professor Xunjing Li. The author thanks both of them for their help.

\section{References}

[1] N. U. Ahmed, "Properties of relaxed trajectories for a class of nonlinear evolution equations on a Banach space", SIAM J. Control Optim. 21 (1983) 953-967.

[2] E. J. Balder, "New existence results for optimal controls in the absence of convexity: the importance of extremality", SIAM J. Control Optim. 32 (1994) 890-916.

[3] L. D. Berkovitz, Optimal control theory (Springer, New York, 1983). 
[4] L. Cesari, "Existence theorem for weak and usual solutions in Lagrange problems with unilateral constraints I", Trans. Amer. Math. Soc. 124 (1966) 369-412.

[5] L. Cesari, Optimization theory and applications, problems with ordinary equations (Springer, New York, 1983).

[6] H. O. Fattorini, Relaxed controls in semilinear infinite dimensional systems, Int. Ser. Num. Math. 100 (Birkhäuser, Basel, 1991) 115-128.

[7] A. F. Filippov, "On certain questions in the theory of optimal control", SIAM J. Control Ser. A, Control 1 (1962) 76-84.

[8] R. Gamkrelidze, Principle of optimal control theory (Plenum Press, New York, 1978).

[9] H. Hermes and J. LaSalle, Functional analysis and time optimal control (Academic Press, New York, 1969).

[10] D. Kinderlehrer and G. Stampacchia, An introduction to variational inequalities (Academic Press, New York, 1981).

[11] X. Li and J. Yong, Optimal control theory for infinite dimensional systems (Birkhäuser, Boston, 1995).

[12] E. C. McShane, "Generalized curves", Duke Math. J. 6 (1940) 513-536.

[13] E. C. McShane, "Relaxed controls and variational problems", SIAM J. Control 5 (1967) 438-485.

[14] C. B. Morrey, Jr., Multiple integrals in the calculus of variations (Springer, Berlin, 1966).

[15] L. W. Neustadt, "The existence of optimal controls in the absence of convexity conditions", J. Math. Anal. Appl. 7 (1963) 110-117.

[16] N. S. Papageorgiou, "Properties of the relaxed trajectories of evolution equations and optimal control", SIAM J. Control Optim. 27 (1989) 267-288.

[17] M. B. Suryanarayana, "Existence theorems for optimization problem concerning linear, hyperbolic partial differential equations without convexity conditions", J. Optim. Theory Appl. 19 (1976) 4761.

[18] J. Warga, "Relaxed variational problems", J. Math. Anal. Appl. 4 (1962) 111-128.

[19] J. Warga, "Functions of relaxed controls", SIAM J. Control 5 (1967) 628-641.

[20] J. Warga, Optimal control of differential and functional equations (Academic Press, New York, 1972).

[21] L. C. Young, "Generalized curves and the existence of an attained absolute minimum in the calculus of variations", C. R. Sci. Lettres Varsovie, C. III 30 (1937) 212-234.

[22] L. C. Young, Lectures on the calculus of variational and optimal control theory (W. B. Saunders, Philadelphia, 1969). 